\title{
Getting Back to the Basics. Parameters That Must Be Considered Before Attempting Quantitative EDS Analysis in the TEM
}

\author{
N. Rowlands ${ }^{1}$, E.F. Schumacher ${ }^{2}$ and A.W. Nicholls ${ }^{3}$ \\ Oxford Instruments Inc, 300 Baker Ave, Concord, MA 01742, USA \\ McCrone Associates Inc, 850 Pasquinelli Drive Westmont, IL 60559-5539 USA \\ University of Illinois at Chicago, 845 West Taylor St., Chicago, IL 60607-7058 USA
}

Good quantitative EDS data are difficult to obtain in the TEM due to many different factors, including the sample, TEM design, hard X-ray shielding, specimen holder and EDS configuration. All of these factors can contribute to whether or not accurate results may be achieved and these parameters may be quite different between TEMs depending upon column design, beam energy, pole piece design, sample holder and detector positioning.

The major impetus in collecting data here is to demonstrate how different geometries and analytical conditions may have considerable effect on the outcome of quantitative results, particularly when analyzing light elements. Data may often be collected in a manner that is not conducive to the rigors needed for accurate analysis. It can also be demonstrated that not only can quantitative EDS results be adversely affected by changing analytical conditions but count rates, peak to background ratios and spectral artifacts may be changed to a considerable extent by changing probe and sample positions within the TEM and sample holder.

Three different TEMs were used in this study to compare the effect of sample holders, beam positioning, tilting and take off angle (TAO). These TEMs varied in age from 2 to 20 years old. Data were collected to demonstrate the effects of the above parameters and therefore how to choose the best conditions for analysis. TEM1 is a modern cold field emission aberration corrected instrument with custom designed low background holders for EDS analysis (TOA 18 ${ }^{\circ}$ ). TEM2 is a 10 year old LaB6 300kV TEM with both low background holder and general purpose holder (TOA $17.5^{\circ}$ ). TEM3 is an historical 200kV TEM approximately 20years old with TOA $11^{\circ}$. All instruments were equipped with an Oxford Instruments X-Max80TEM SDD.

Results show significant differences between instruments, especially in the case of the older TEM (Figure 2) where tilting is necessary to obtain meaningful results for quantification, especially if light element quant is required. However, there appears to be attenuation of light elements in all systems to varying extents.

Although results on TEMs1 and 2 show less attenuation than TEM3 when data are collected at the grid center, the comparison of oxygen concentration between center and grid edge show that attenuation is a very real problem for low energy peaks (Figure 3). It is, therefore, most important to take into account positioning of the analytical probe when attempting to quantify elements in TEM, especially when using low energy lines. 


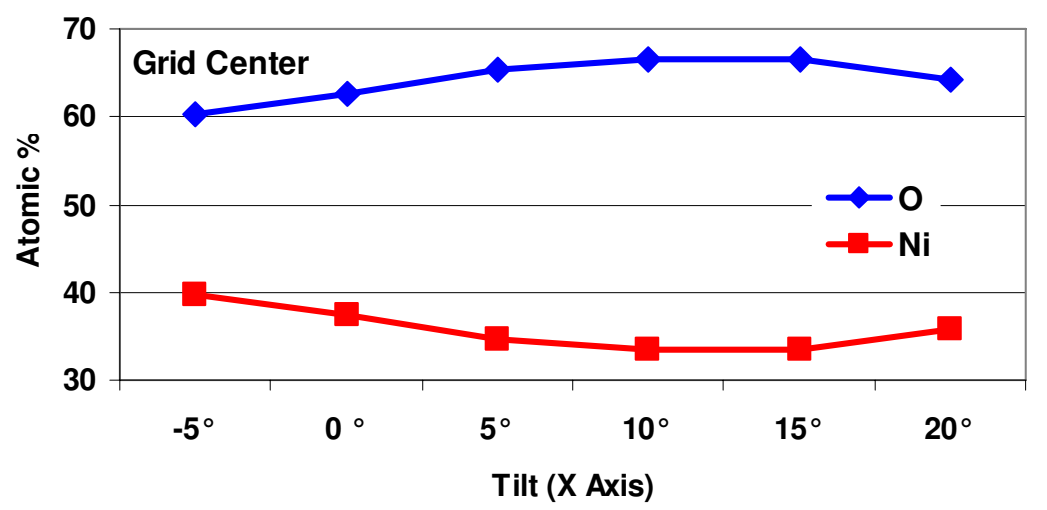

Figure1. Effect of tilting sample in TEM1 showing changes in concentration of $\mathrm{Ni}$ and $\mathrm{O}$. There is a slight increase in $\mathrm{Ni} / \mathrm{O}$ ratios from -5 to 10 degree tilt.

Figure2. Effect of tilting sample in TEM3 showing larger changes in concentration of $\mathrm{Ni}$ and $\mathrm{O}$ and increase in overall $\mathrm{X}$ ray count rate between 0 and 15 degrees tilt, due to the shallower TOA and the sample holder design.
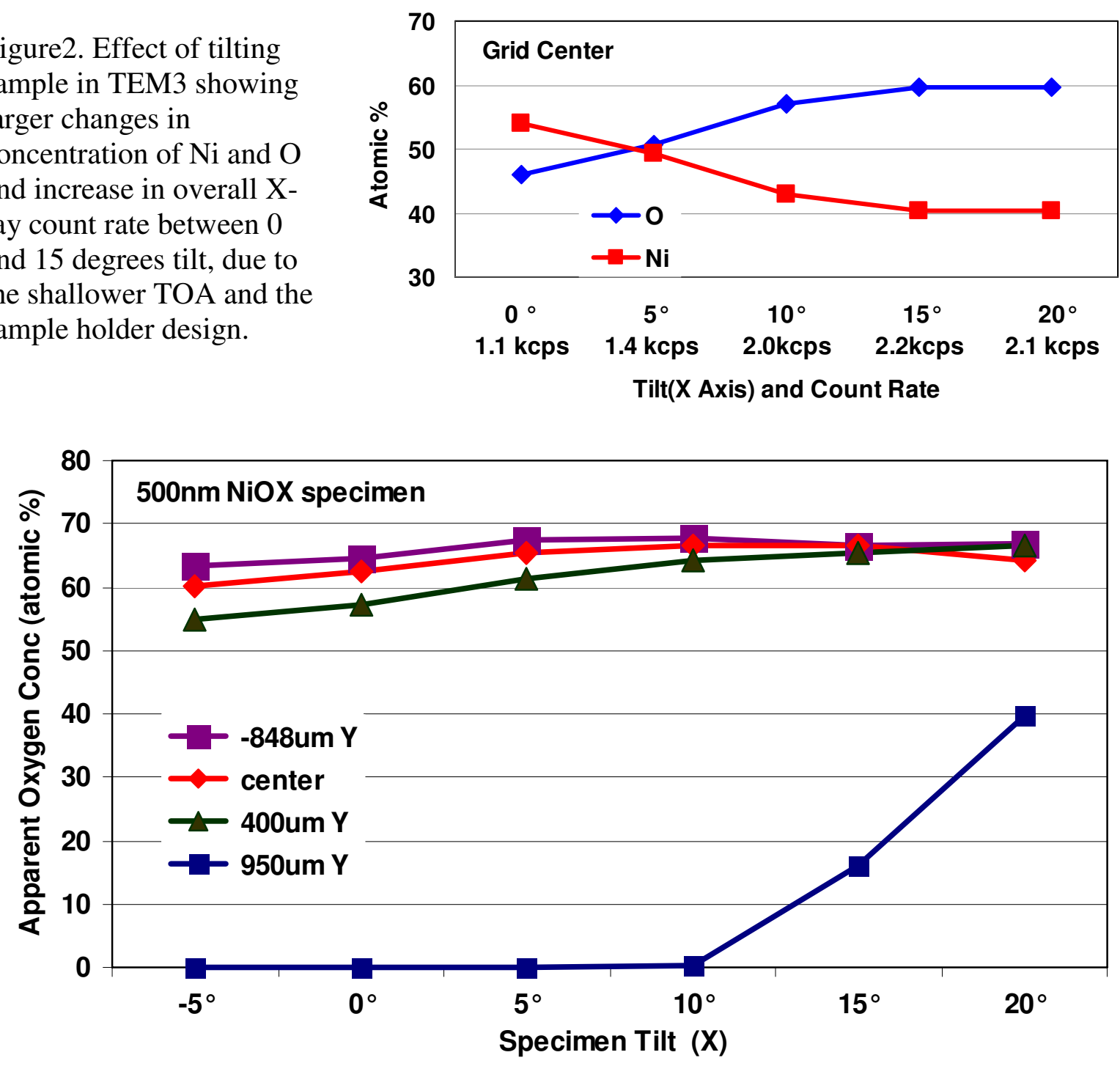

Figure3. Effect of sample position and tilt, on apparent oxygen concentration of a NiOX specimen, in TEM1. Positive Y shift is moving closer to the X-ray cut out in the holder. 